\title{
Self-Control Demands and Job Strain in Nursing and Care: The Moderating Role of Boundary Conditions
}

\section{Klaus-Helmut Schmidt}

Leibniz Research Centre for Working Environment and Human Factors, Technical University of Dortmund, Germany

*Corresponding author: Klaus-Helmut Schmidt, Leibniz Research Centre for Working Environment and Human Factors, Technical University of Dortmund, Ardeystr. 67 , Dortmund, Germany; Tel: 00492311084327; E-mail: schmidtkh@ifado.de

Received date: Feb10, 2015, Accepted date: Apr 01, 2015, Published date: Apr 08, 2015

Copyright: $\odot 2015$ Schmidt $\mathrm{KH}$. This is an open-access article distributed under the terms of the Creative Commons Attribution License, which permits unrestricted use, distribution, and reproduction in any medium, provided the original author and source are credited.

\begin{abstract}
Recent theoretical developments and findings in basic research suggest that, self-control demands are a unique job stressor. A series of studies among healthcare workers are reported corroborating this view. The results of literature review show that different forms of self-control demands such as impulse control, resisting distractions, overcoming inner resistances contribute significant portions of unique variance to the prediction of various indicators of job strain and interact with other forms of self-control demands. Furthermore, the relationship between self-control demands and strain is moderated by various resources like self-control capacity, affective organizational commitment, and job control. The identification of those resources offers promising starting points for the development of preventive interventions.
\end{abstract}

Keywords: Control resource; Job strain; Self-control strength; Wellbeing

\section{Introduction}

Self-control demands are an integral component of people works [1] requiring employees to suppress spontaneous impulsive response tendencies and associated affect states in order to follow certain organizationally desired 'display rules' and to create role-compatible impressions. For example, the role of healthcare workers usually involves the requirement of showing empathy, helpfulness, and patience, but they are more or less frequently faced with situations eliciting frustration, anger, or even aggression and aversion. The meeting of self-control demands is an important constituent of nursing and care [2].

According to a widespread notion, self-control can be defined as overriding or inhibiting automatic, habitual, or spontaneous action tendencies, urges, emotions or desires that would otherwise interfere with purposeful, goal-directed behavior [3]. Thus, demands on selfcontrol cause people to change the way they would spontaneously think, feel, or behave.

Although, self-control is related to personal success in many domains of life [4], a growing body of evidence in basic research strongly suggests that exercising self-control is associated with psychological costs, which are manifested as impaired performance and psychological strain [5]. In contrast to this expanding line of basic research, aspects of self-control have only recently received attention in the literature on job-related stress and health. The main aim of this review is to outline and summarize the first steps of applying the concept of self-control to real work settings in general and healthcare work in particular. The job-related application of self-control research includes; the development of an instrument to assess self-control demands in service professions, the analyses of adverse effects of those demands on healthcare workers' psychological well-being and health, and the identification of boundary conditions, which might moderate (i.e., strengthen or weaken) the relationship of the stressor with indicators of job strain and well-being. The review begins with a brief summary of the empirical evidence from basic research on the costs of self-control. Then, the relationship between job-related selfcontrol demands and job strain will be discussed, including some boundary conditions, which moderate this relationship.

\section{Psychological Costs of Self-Control}

Findings on self-control in basic research show that exercising selfcontrol can lead to impairments in cognitive and behavioral control and causes psychological strain [6]. In a series of experimental studies that demanded two successive acts of self-control, self-control performance on the second act was consistently impaired. The impairment was found even when quite different domains of selfcontrol were involved [5]. Acts of self-control involved regulating emotions and affective states, suppressing spontaneous and habitual impulses, overcoming inner motivational resistances, resisting interfering distractions, and updating working memory. In the experimental studies, acts of self-control were found to cause increases in self-reported effort, tiredness, and exhaustion [6], as well as increases in blood pressure and heart rate variability indicating typical stress responses $[7,8]$.

A model of self-control was proposed to account for these observations [3]. According to this model, different forms of selfcontrol draw on a common regulatory resource, or self-control strength, which is limited and depleted in the process of exerting selfcontrol. Consequently, acts of self-control reduce the strength available for subsequent self-control efforts. The term 'ego depletion' was coined to describe this state of diminished self-control strength [3]. Furthermore, the model suggests that people who frequently need to exert self-control without being able to replenish their self-control strength are likely to fall into a state of chronic resource depletion and, as a result, suffer from chronically high psychological strain and impaired well-being. 
Page 2 of 6

Inspired by this model, a variety of forms of self-control behavior in everyday life has also been found to draw on and deplete the limited control resource such as managing one's impression and selfpresentation, suppressing stereotypes and prejudices, restraining anger and aggression, managing emotions, or overcoming unwanted impulses [3]. Despite the growing body of evidence on everyday life self-control and its potential role as a source of stress, self-control demands have only recently received attention in the literature on jobrelated stress and health in general, and stress and health of healthcare workers in particular.

\section{Self-Control Demands as a Source of Stress at Work}

To provide insights into the role of self-control demands in work settings, a self-report instrument was developed focusing on three forms of job-related self-control demands [9]. First, impulse control refers to the demand to inhibit spontaneous, impulsive response tendencies and affective states associated with injudicious expressions (example items: 'My job requires me never to lose my temper', 'I am never allowed to become impatient at work'). Second, resisting distractions involves the requirement to ignore or resist distractions evoked by task-irrelevant stimuli, which would otherwise interfere with a successful accomplishment of tasks (example items: 'If I want to get my work done successfully, I must not give in to any distractions', 'My work requires me to resist distractions'). Third, overcoming inner resistance relates to the requirement to overcome motivational deficits to complete unattractive tasks that cannot be postponed or evaded (example items: 'Some of my tasks are such that, I really need to force myself to get them done', 'Dealing with unattractive tasks requires of me a high amount of will-power').

The three scales have been repeatedly shown to cover factorially distinct, moderately correlated forms of job-related self-control demands $[10,11]$. In a longitudinal study, the measured self-control demands were found to be very stable over 12 and 24 months, indicating that they are stable characteristics of a given job [11]. Similarly, the results from studies among different service providers showed that after controlling biographical and sample attributes, selfcontrol demands explained additional variance in indicators of job strain over and beyond that accounted for by other established work stressors, like workload, role stress, and lack of social support $[9,12]$. Finally, self-control processes have been proposed and found to be significant mediators in the relationship of other well-known stressors (such as time pressure and concentration requirements) and job strain and impaired well-being [13-15]. This observation is in line with Action Regulation Theory [16], which suggests that, those stressors exert their adverse influences because they disturb goal-directed behavior and therefore demand from employees, to volitionally control cognitive, emotional, and behavioral processes.

The relationship between self-control demands and job strain and impaired well-being raises the question as to whether factors could be identified in the work environment or in the person that might strengthen or weaken the adverse influences of self-control demands. The identification of such boundary conditions or moderators is a dominant topic in stress research, not at least due to their implications for job redesign, training, or personal selection.

\section{Effect-Strengthening Boundary Conditions}

\section{Emotional Labor}

Emotional labor refers to the goal-directed regulation and expression of organizationally desired emotions [17]. Emotional labor has repeatedly been found to act as a source of work stress and to cause strain (e.g., burnout), especially when emotional dissonance is experienced [18-20]. The experience of emotional dissonance does emerge when emotions have to be displayed, which are not truly felt.

Several scholars have considered the adverse effects of emotional dissonance from the perspective of self-control [20,21]. Accordingly, portraying emotions contrary to one's genuinely felt emotions is a form of response-focused emotion regulation [22]. This regulation strategy is associated with efforts to control emotional expressions as required (e.g., suppressing felt emotions or displaying required emotions exaggeratedly) after an emotional response has been triggered. Emotional dissonance has been demonstrated to cause people to exert response-focused emotion regulation, which elicits high sympathetic arousal and results in psychological strain [7]. This finding led scholars to conceptualize response-focused emotion regulation as an act of self-control that resolves the discrepancy between emotions felt and required emotions [23]. Hence, emotional dissonance was hypothesized to exert its adverse influences on job strain through the depletion of a limited regulatory resource [20].

In line with this reasoning, it has been proposed that if coping with emotional dissonance involves exerting self-control in the form of response-focused emotion regulation, and thus, consumes a limited regulatory resource, emotional dissonance and self-control demands can be expected to exert interactive effects on job strain [24]. The positive relation of one stressor to strain should be amplified as a function of the other stressor. This proposition builds on the argument that, emotional dissonance draws on and depletes the same resource as other self-control demands do.

A study among healthcare workers of a municipal organization for residential elderly care confirmed the predictions [24]. The relationship between self-control demands and emotional exhaustion (the core symptom of burnout) was positive and significantly stronger when emotional dissonance was high than when emotional dissonance was low. A similar interaction pattern emerged in a measure of depressive symptoms and a measure of absence behavior.

\section{Goal Discrepancy}

The perceived discrepancy between personal and organizational goals can be conceptualized as a form of self-control demands and similar to emotional dissonance - should strengthen the adverse influence of situational self-control demands. Traditionally being linked to the person-environment (P-O) fit literature, goal congruence is defined as the degree of fit between personal and organizational goals $[25,26]$. In healthcare settings, for instance, employees often experience a mismatch between their personal goals of helping other people or providing high quality care and the economical goals and bureaucratic demands of the organization [27,28]. Recent metaanalyses have demonstrated consistently that an increasing mismatch between personal and organizational goals goes along with a decrease in job satisfaction and organizational commitment as well as increasing intentions to quit $[27,28]$. For explaining the adverse effects of incongruent personal and organizational goals, it has been argued that incongruent goals may lead to psychological conflict and that a 
resolution of this conflict requires self-control efforts consuming and depleting a limited control resource [29]. Since employees usually try to achieve organizational goals because they expect to receive positive outcomes in return, they resolve the conflict by suppressing their personal goals and simultaneously compensating their insufficient extrinsic motivation for pursuing the goals of the organization. This kind of conflict resolution is a form of self-control that, in more general terms, can be conceptualized as overriding or inhibiting automatic or habitual action tendencies or desires in order to ensure an unimpaired realization of purposeful behavior [30,31].

The notion that the adverse influences of incongruent personal and organizational goals are due to conflict-resolving self-control efforts suggests that perceived goal discrepancies should interact with situational self-control demands (such as impulse control or resisting distractions) in predicting psychological strain [29]. The rationale behind this prediction is that both stressors (goal incongruence and situational self-control demands) draw on and compete for the same limited control resource.

Data collected among a sample of elderly care nurses confirmed this prediction [32]. In this study, participants were asked to rank eight different goals first according to their own personal priorities (personal goals), and second according to the priorities of the organization as perceived by the participants (perceived organizational goals). Half of the goals were economical in nature, the other half focused on humanitarian concerns. In line with the expection, it was observed that the adverse influences of increasing situational selfcontrol demands were boosted with increasing goal discrepancies. The predicted interactive relationship did emerge in both self-report measures of strain (burnout, psychosomatic complaints) and a measure of absenteeism [32].

\section{Cognitive Control Deficits}

A growing body of evidence on self-regulatory functioning suggests that, daily cognitive control deficits in the form of frequent failures in perception, action, self-regulation, and affective control represent a valid, though distal indicator for the individual capacity of the control resource [33]. For example, specific concentration problems, like the inability to be focused during a conservation, as well as emotion control problems, like affective huffiness or injudicious expressions, are typical behavioral manifestations of control deficits, which are frequently measured with a self-report instrument [34]. A high intraindividual stability of cognitive control deficits has repeatedly been observed and most researchers concur with the notion that these deficits constitute a person-related trait reflecting interindividual differences in self-control strength [33].

If this notion is valid, then employees with high control deficits can be expected to be more susceptible to the adverse influences of jobrelated self-control demands and, thus, experience higher levels of strain than those with low deficits. This is corroborated with study [35]. For employees with a high level of control deficits, the adverse impact of job-related self-control demands was more pronounced than for employees suffering less from control deficits.

\section{Emotional Labor and Cognitive Control Deficits}

In coping with emotional dissonance as the experienced discrepancy between genuinely felt and organizationally desired emotions, two strategies can be used [17]. Surface acting, the first strategy involves the regulation of emotional expression with the aim of bringing it in line with organizational display rules, whereas deep acting (the second strategy) aims at modifying the situation or perception of situation in order to change felt emotions according to external display rules. Consequently, surface acting as a responsefocused form of emotional regulation leaves genuinely felt emotions unchanged, whereas engaging in deep acting as an antecedent-focused form of emotional regulation makes required and felt emotions agree.

Two recent meta-analyses revealed substantial positive relationships between surface acting and impaired personal and jobrelated well-being and a negative relationship with performance outcomes [36,37]. In contrast to this, deep acting was found to be only weakly related to impaired well-being, but positively associated with emotional performance and customer satisfaction [36,37]. In nursing settings similar differential relationships of both emotional labor strategies have been identified. Among samples of nurses, surface acting was observed to be positively linked to burnout and negatively linked with job satisfaction. Deep acting showed weaker relationships with burnout and no associations with nurses' job satisfaction [38-40].

To advance our understanding of the differential relationships of both emotional labor strategies and drawing on the strength model of self-control [3], several scholars have argued that engaging in surface acting puts higher demands on self-control resources than engaging in deep acting and thus, depletes resources to a greater extent $[41,42]$. Whereas surface acting involves the continuous monitoring of genuinely felt and required emotions combined with the continuous investment of mental effort to alter ones emotional expression, deep acting calls for control resources only at the onset of an emotion by deploying attention or reappraising situations in order to induce the desired emotion $[43,44]$.

Since the resource demands of surface and deep acting cannot be directly assessed, a recent study [45] adopted a moderation approach to provide evidence for the differential regulatory features of both emotional labor strategies. The moderator approach integrates the argument of differential resource demands of surface and deep acting and the assumption of a limited individual capacity for self-control. On the basic of the integration of both arguments, it was predicted that the limited individual control capacity (indicated by control deficits) should exert a stronger moderating influence on the relationship of surface acting to well-being and health than on the corresponding relationship of deep acting.

Among a sample of nurses employed at one hospital and three nursing homes, the study revealed significant positive relationships between surface acting and cognitive control deficits with indicators of job strain, whereas deep acting was unrelated to strain [45]. In line with expectations, surface acting was found to interact stronger with cognitive control deficits in its relationship to strain than deep acting. Compared to nurses with low levels of cognitive deficits, the adverse influences of surface acting on burnout, depressive symptoms, and absenteeism were much stronger when high levels of cognitive control deficits were reported [45].

In addition to the effect-strengthening boundary conditions, some moderates have been identified that contribute to a weakening of the adverse effects of job-related self-control demands. Such kinds of boundary conditions are often labelled as 'psychological resources' [13]. 


\section{Effect-Weakening Boundary Conditions}

\section{Self-Control Capacity}

Complementary to cognitive control deficits as a personal vulnerability factor (and distal indicator of the individual capacity for self-control), more proximal and direct measures of that kind of capacity can be expected as well to moderate the detrimental influences of self-control demands. A self-report instrument allows getting immediate access to assess the individual capacity for selfcontrol [46]. The scale addresses various domains of self-control, such as control of thoughts, emotions, impulses, and performance. Similar to the measure of control deficits, the trait measure of self-control capacity was found to be one-dimensional in nature and has high intraindividual stability [46].

Based on the assumptions that self-control demands are a source of stress at work drawing on and depleting a limited regulatory resource, and people differs regarding their personal self-control capacity, selfcontrol capacity can be expected to interact with self-control demands in predicting job strain and well-being. Specifically, the adverse impact of self-control demands should be attenuated as a function of increasing levels of self-control capacity. The theoretical rationale behind this prediction is that employees with high levels of selfcontrol capacity should have a greater resource at their disposal to cope with self-control demands.

A recent study among healthcare workers [47] provided support for this prediction. Emotional exhaustion, depressive symptoms, and sleep disorders (as strain outcomes) did reflect interactive influences of selfcontrol demands and self-control capacity in such a way that, the adverse effects of self-control demands were weakened with increasing levels of control capacity [47].

\section{Affective Organizational Commitment}

Given the adverse effects of self-control demands, recent studies have focused on mechanisms, which might have the potential to prevent ego depletion, when exerting self-control [48]. Since the control resource obviously does not remain depleted forever, people can recover from ego depletion and replenish their self-control strength. The Broaden- and Build Theory of positive emotions [49], which states that positive emotions broaden people's thought-actionrepertoires and thus facilitate coping with stress, suggests the assumption that positive emotions may help people regain their selfcontrol strength. Results from a series of experimental studies clearly confirmed this notion. After an initial act of self-control, participants who watched a comedy video or received a surprise gift performed just well on various self-control tasks as non-depleted participants and significantly better than participants who experienced a sad mood, a neutral mood, or a brief rest period [48].

The observed lab findings on the beneficial function of positive mood and emotions, led researchers to suggest that affective organizational commitment - a work-related attitude with strong affective roots could play a similar buffering role in work contexts [50]. A study among a sample of nurses provided evidence for this notion. The findings revealed positive relationships of self-control demands and negative relationships of affective organizational commitment with a broad spectrum of strain indicators. In addition, the results confirmed the buffer hypothesis of commitment. The positive relationships between self-control demands and burnout, complaints, turnover intentions, and days absent are attenuated as a function of affective commitment [50].

\section{Job Control}

The Job Demands-Control Model of job strain suggests that job control should function as a potential situational resource, which may protect employees against the detrimental effects of self-control demands [51]. According to this model, the degree of control employees have over their tasks and behaviors while performing their daily work is hypothesized to buffer or moderate the adverse effects of high job demands.

Employees who are faced with self-control demands may profit in a particular way from high situational control opportunities at work. Because high levels of situational control offer employees the chance to prevent external events from being a disturbing distractor or to start with challenging tasks only when they are in a state of high selfcontrol strength. Several studies could demonstrated that for employees who reported low levels of control, self-control demands were positively associated with emotional exhaustion, psychosomatic complaints, and absenteeism as well as negatively related to job satisfaction. By way of contrast, there were less adverse effects of self-control demands on job strain for those who perceived high levels of job control $[52,53]$. Quite similar buffering effects of control were found in the relationship between emotional dissonance as stressor and burnout [54].

\section{Theoretical Implications}

Main aim of the present paper was to outline and summarize research efforts on applying the concept of self-control to work settings, especially nursing and care. Inspired by novel theoretical developments and empirical findings in basic research, self-control demands were suggested to be an unique, yet often neglected job stressor. In line with this reasoning, self-control demands in the form of impulse control, resisting distractions, and overcoming inner resistances jointly contributed significant portions of variance to the prediction of various measures of psychological strain and well-being $[11,12]$.

Furthermore, and on the basis of the theoretical notion that different forms of self-control demands draw on and deplete a common limited control resource, self-control demands were hypothesized and found to interact in the prediction of job strain. The positive relationship between one form of self-control demands and strain was amplified as a function of another simultaneously occurring form of self-control demands. Similar interactive effects resulted from the combination of self-control demands with emotional dissonance on the one hand and with perceived incongruencies between personal and organizational goals on the other [24,32]. These observations suggest that both emotional dissonance and incongruent goals lead nurses to exert selfcontrol, which depletes and overtaxes a limited resource.

The reviewed studies are characterized by various features, which deserve attention. The studies based on cross-sectional and longitudinal data, used a broad spectrum of measures of strain and well-being as outcomes, and used samples from different nursing and caring settings. These features do not only contribute to the generalizability of the observed relationships, but strengthen as well their validity and allows causal inferences. 


\section{Practical Implications}

The present findings have some practical implications for service employees in general, and healthcare workers in particular. Healthcare workers, who are often confronted with high job-related self-control demands are, as demonstrated, at risk of high psychological job strain and impaired well-being. Since self-control demands are an integral constituent of nursing and caring, and cannot be reduced immediately, other strategies are needed to counter the adverse effects of dealing with high self-control demands.

The identified boundary conditions, especially those that weaken or buffer the relationships of self-control demands with job strain, offer promising starting points for developing preventive strategies and interventions. The buffering effect of affective organizational commitment [50] suggests practical measures of creating work environments in such a way that healthcare workers can feel affectively commitment to their organizations. The most effective antecedents of commitment may help to derive corresponding measures. Consequently, perceived organizational support can be assumed to have the strongest beneficial impact on the development of healthcare workers' affective commitment to their organizations [55]. Organizations that need affectively committed employees must demonstrate their own commitment by providing a supportive work environment.

Creating a supportive work environment can help nurses and care givers increase their control over demands they are faced with every day. In such a work environment care givers being temporally unable to meet their demands could ask colleagues to take over some of their workload until they are able to effectively deal with that demands for themselves. Furthermore, organizational strategies such as job redesign or simplifying the paperwork can be used to increase control in nursing and caring professions.

The current findings also show that meeting emotional labor demands is especially straining when nurses tend to adopt surface acting as preferred strategy and when they have low control resources at their disposal [45]. Consequently, interventions are needed to inform nurses on the detrimental effects of surface acting and train them on the daily use of deep acting as the healthier emotional regulation strategy. Such interventions seem to be promising as both emotional labor strategies are not stable, trait-like individual characteristics but can rather be adopted flexibly [56].

Interventions targeted at the enhancement of the resource capacity for self-control have been found to be effective [47]. One of such intervention had participants enter a two-month physical exercise programme that was intended to improve their self-control [57]. After the programme, participants showed considerable improvements in laboratory self-control tasks. Participants also reported significant decreases in perceived stress, emotional distress, smoking, alcohol and caffeine consumption, an increase in healthy eating, emotional control and attendance to commitments. The idea that the capacity for selfcontrol can be improved is of considerable practical importance, not at least in the field of nursing and caring.

Finally, the effect-strengthening boundary condition of perceived incongruencies between personal and organizational goals suggests interventions aiming at reducing resulting goal conflicts [32]. One strategy could be that the management of healthcare organizations expresses a clear commitment to the mission of caring and at the same time win their nursing staff for an efficient use of resources in following that mission. This doubtlessly requires a thoughtful communication of all goals being of vital importance to nursing and caring. As a result of these efforts, staff can be made ready to integrate all relevant organizational goals into their own personal goal systems [32].

\section{Summary}

Self-control demands represent an unique and often neglected job stressor, which draws on and depletes limited control resources. As suggested by the results of our studies on boundary conditions of the relationship between self-control demands and job strain, strengthening resources such as job control, affective organizational commitment, and self-control capacity may protect healthcare workers against the adverse effects of coping with self-control demands.

\section{References}

1. Brotheridge CM, Grandey AA (2002) Emotional labor and burnout: comparing two perspectives of "people work". J Vocat Behav 60: 17-39.

2. Cohen-Mansfield J (1995) Stress in nursing home staff: A review and a theoretical model. J ApplGerontol 14: 444-466.

3. Baumeister RF, Vohs KD, Tice DM (2007) The strength model of selfcontrol. CDPS 16: 351-355.

4. Baumeister RF, Vohs KD (Eds.) (2004) Handbook of self-regulation. Research, theory, and applications. New York: Guilford Press.

5. Hagger MS, Wood C, Stiff C, Chatzisarantis NL (2010) Ego depletion and the strength model of self-control: a meta-analysis. Psychol Bull 136: 495-525.

6. Muraven M, Tice DM, Baumeister RF (1998) Self-control as limited resource: regulatory depletion patterns. J Pers Soc Psychol 74: 774-789.

7. Robinson JL, Demaree HA (2007) Physiological and cognitive effects of expressive dissonance. Brain Cogn 63: 70-78.

8. Segerstrom SC, Nes LS (2007) Heart rate variability reflects selfregulatory strength, effort, and fatigue. PsycholSci 18: 275-281.

9. Schmidt KH, Neubach B (2007) Selbstkontrollanforderungen: Einevernachlässigtespezifische Belastung squellebei der Arbeit. ZblArbeitsmed 57: 342-348.

10. Schmidt KH, Neubach B (2009) Selbstkontroll anforderungenalsspezifischeBelastung squellebei der Arbeit [Self-control demands as a specific source of stress at work]. Z Personalpsychol 8: 169-179.

11. Schmidt KH, Neubach B (2010) Selbstkontrollanforderungenbei der Arbeit

FragebogenzurErfassungeinesbislangwenigbeachtetenBelastungsfaktors [Self-control demands-questionnaire for measuring a so far neglected job stressor]. Diagnostica 56: 133-143.

12. Schmidt KH, Neubach B (2007) Self-control demands: A source of stress at work. Int J Stress Management 14: 398-416.

13. Hobfoll SE (2002) Social and psychological resources and adaptation. Rev General Psychol 6: 307-324.

14. Diestel S, Schmidt KH (2009) Mediator and moderator effects of demands on self-control in the relationship between work load and indicators of job strain. Work \& Stress, 23: 60-79.

15. Diestel S, Schmidt KH (2012) Lagged mediator effects of self-control demands on psychological strain and absenteeism. J Occup Organiz Psychol 85: 556-578.

16. Hacker W (2003) Action Regulation Theory: A practical tool for the design of modern work processes? Eur J Work Organiz Psychol 12: 105-130.

17. Hochschild A (1983) The managed heart. Berkeley, CA: University of California Press.

18. Abraham R (1998) Emotional dissonance in organizations: Antecedents, consequences, and moderators. Genet Soc General Psychol Monogr 124: $229-246$. 
19. Heuven E, Bakker AB (2003) Emotional dissonance and burnout among cabin attendants. Eur J Work Organiz Psychol 12: 81-100.

20. Zapf D, Holz M (2006) On the positive and negative effects of emotion work in organizations. Eur J Work Organiz Psychol 15: 1-28.

21. Judge TA, Woolf EF, Hurst C (2009) Is emotional labor more difficult for some than for others? A multilevel, experience-sampling study. Pers Psychol 62: 57-88.

22. Gross JJ (2001) Emotion regulation in adulthood: Timing is everything. CDPS 10: 214-219.

23. Schmeichel BJ, Vohs KD, Baumeister RF (2003) Intellectual performance and ego depletion: role of the self in logical reasoning and other information processing. J Pers Soc Psychol 85: 33-46.

24. Diestel S, Schmidt KH (2011) Costs of simultaneous coping with emotional dissonance and self-control demands at work: results from two German samples. J Appl Psychol 96: 643-653.

25. Verquer ML, Beehr TA, Wagner SH (2003) A meta-analysis of relations between person-organization fit and work attitudes. J Vocat Behav 63: 473-489.

26. Kristof-Brown AL, Zimmerman RD, Johnson EC (2005) Consequences of individuals fit at work: a meta-analysis of person-job, personorganization, person-group, and person-supervisor fit. Pers Psychol 58: 281-342.

27. Takase M, Maude P, Manias E (2005) Explaining nurses' work behaviour from their perception of the environment and work values. Int J Nurs Stud 42: 889-898.

28. Daehlen M (2008) Job satisfaction and job values among beginning nurses: a questionnaire survey. Int J Nurs Stud 45: 1789-1799.

29. Kehr HM (2004) Integrating implicit motives, explicit motives, and perceived abilities: The compensatory model of work motivation and volition. AMR 29: 479-499.

30. Baumeister RF, Heatherton TF, Tice DM (1994) Losing control: How and why people fail at self-regulation. San Diego: Academic Press.

31. Schmeichel BJ (2007) Attention control, memory updating, and emotion regulation temporarily reduce the capacity for executive control. J ExpPsychol Gen 136: 241-255.

32. Schmidt KH (2010) The relation of goal incongruence and self-control demands to indicators of job strain among elderly care nursing staff: A cross-sectional survey study combined with longitudinally assessed absence measures. Int J Nurs Stud 47: 855-863.

33. Larson GE, Alderton DL, Neideffer M, Underhill E (1997) Further evidence on dimensionality and correlates of the Cognitive Failures Questionnaire. Br J Psychol 88: 29-38.

34. Broadbent DE, Cooper PF, FitzGerald P, Parkes KR (1982) The Cognitive Failures Questionnaire (CFQ) and its correlates. Br J Clin Psychol $21: 1-16$

35. Schmidt KH, Neubach B, Heuer H (2007) Self-control demands, cognitive control deficits, and burnout. Work \& Stress 21: 142-154.

36. Hülsheger UR, Schewe AF (2011) On the costs and benefits of emotional labor: a meta-analysis of three decades of research. J Occup Health Psychol 16: 361-389.

37. Mesmer-Magnus JR, De Church LA, Wax A (2012) Moving emotional labor beyond surface and deep acting: a discordance-congruence perspective. OrganizPsychol Rev 2: 6-53.

38. Diefendorff JM1, Erickson RJ, Grandey AA, Dahling JJ (2011) Emotional display rules as work unit norms: a multilevel analysis of emotional labor among nurses. J Occup Health Psychol 16: 170-186.
39. Pisaniello SL, Winefield HR, Delfabbro PH (2012) The influence of emotional labour and emotional work on the occupational health and wellbeing of South Australian hospital nurses. J Vocat Behav 80: 579-591.

40. Chou HY, Hecker R, Martin A (2012) Predicting nurses' well-being from job demands and resources: a cross-sectional study of emotional labour. J Nurs Manag 20: 502-511.

41. Martínez-Iñigo D, Totterdell P, Alcover CM, Holman D (2007) Emotional labour and emotional exhaustion: Interpersonal and intrapersonal mechanisms. Work \& Stress 21: 30-47.

42. Totterdell P, Holman D (2003) Emotion regulation in customer service roles: testing a model of emotional labor. J Occup Health Psychol 8: 55-73.

43. Grandey AA (2000) Emotion regulation in the workplace: a new way to conceptualize emotional labor. J Occup Health Psychol 5: 95-110.

44. Gross JJ (1998) The emerging field of emotion regulation: An integrative review. Rev General Psychol 2: 271-299.

45. Schmidt KH, Diestel S2 (2014) Are emotional labour strategies by nurses associated with psychological costs? A cross-sectional survey. Int J Nurs Stud 51: 1450-1461.

46. Tangney JP1, Baumeister RF, Boone AL (2004) High self-control predicts good adjustment, less pathology, better grades, and interpersonal success. J Pers 72: 271-324.

47. Schmidt KH, Hupke M, Diestel S (2012) Does dispositional capacity for self-control attenuate the relation between self-control demands at work and indicators of job strain? Work \& Stress 26: 21-38.

48. Tice DM, Baumeister RF, Shmueli D, Muraven M (2007) Restoring the self: Positive affect helps improve self-regulation following ego depletion. JESP 43: 379-384.

49. Fredrickson BL, Joiner T (2002) Positive emotions trigger upward spirals toward emotional well-being. Psychol Sci 13: 172-175.

50. Schmidt KH, Diestel S (2012) The relation of self-control demands to job strain: the moderating role of organisational commitment. ApplPsychol: Int Rev 61: 479-497.

51. Karasek R (1979) Job demands, job decision latitude and mental strain: Implications for job redesign. ASQ 24: 285-308.

52. Neubach B, Schmidt KH (2006) Beanspruchungswirkungen von Selbstkontrollanforderungen und Kontrollmöglichkeitenbei der Arbeit. [Effects of self-control demands and job control on occupational strain]. Z Psychol 214: 150-160.

53. Schmidt KH, Diestel S (2011) Differential effects of decision latitude and control on the job demands-strain relationship: A cross-sectional survey study among elderly care nursing staff. Int J Nurs Stud 48: 307-317.

54. Freund N, Diestel S, Schmidt KH (2012) Kontrollspielräumeals protektive Ressourcebei Emotionsarbeit. Z Arbeits Organisations psychol 56: $143-151$

55. Meyer JP, Stanley DJ, Herscovitch L, Topolnytsky L (2002) Affective, continuance, and normative commitment to the organization: A metaanalysis of antecedents, correlates, and consequences. J Vocat Behav 61: 20-52.

56. Philipp A, Schüpbach H (2010) Longitudinal effects of emotional labour on emotional exhaustion and dedication of teachers. J Occup Health Psychol 15: 494-504.

57. Oaten M, Cheng K (2006) Improved Self-Control: The benefits of a regular program of academic study. BASP 28: 1-16. 